\title{
Índice de vulnerabilidad sísmica de escuelas del Área Metropolitana de Medellín, Colombia
}

\author{
- Faver Norbey Zora MejÍA ${ }^{1}$ \\ ANA BEATRIZ ACEVEdo JARAMILLO ${ }^{1}$
}

\section{Resumen}

En este trabajo se presenta la evaluación de la vulnerabilidad sísmica de un inventario de escuelas ubicadas en las ciudades de Medellín, Itagüí y Sabaneta, Colombia, a través de la aplicación del método del Índice Prioritario desarrollado por Hassan y Sözen (1997). El procedimiento del Índice Prioritario permite identificar, de un grupo de estructuras de pórticos de hormigón reforzado, aquellas que podrían sufrir daño severo o colapso en caso de un evento sísmico importante y, por lo tanto, requieren de una evaluación de vulnerabilidad sísmica detallada. Se evaluaron un total de 82 estructuras pertenecientes a 30 instituciones educativas. El sistema de resistencia a cargas laterales de todas las estructuras evaluadas corresponde a pórticos de hormigón reforzado con presencia de muros de mampostería. La aplicación del método del Índice Prioritario identificó un 61\% de las estructuras analizadas como prioritarias, es decir, con posibilidad de que sufran daño severo o colapso ante un futuro evento sísmico. En el desarrollo de esta investigación se propuso incluir parámetros adicionales al método para la evaluación de las estructuras: año de construcción y presencia de columnas cortas. Finalmente, se identificó un 60\% de las estructuras con prioridad alta; este alto porcentaje implica que una gran cantidad de menores estarían en alto riesgo ante la ocurrencia de un evento sísmico.

Palabras clave: Riesgo sísmico, vulnerabilidad sísmica, hormigón reforzado, escuelas.

1 Universidad Eafit. Medellín, Colombia.

Autor de correspondencia: Zora Mejía, F.N. (Faver Norbey): Calle 25A\#58D74, Cabañas-Bello. Teléfono: 3185200630.

Correo electrónico: fzoramej@eafit.edu.co
Historia del artículo:

Artículo recibido: 9-XI-2016/ Aprobado: 30-V-2019

Disponible online: 3 de julio de 2019

Discusión abierta hasta marzo de 2021 


\title{
Seismic Vulnerability Index Of Schools of The Metropolitan Area Of Medellin, Colombia
}

\begin{abstract}
This paper presents the seismic vulnerability assessment of a group of schools located in the cities of Medellín, Itagüí and Sabaneta, Colombia, by the use of the Priority Index methodology developed by Hassan and Sözen (1997). The Priority Index allows the identification, among a group of reinforced concrete structures, of those that could suffer severe damage or collapse due to an important seismic event. A details seismic vulnerability assessment should be applied to the identified structures. A total of 82 structures from 30 schools were evaluated in this work. The lateral load resisting system of all of the structures corresponds to reinforced concrete frames with masonry infill. The application of the Priority Index method classified $61 \%$ of the analyzed structures with high priority, that is, there is a possibility for those structures to suffer severe damage of collapse in a seismic event. Additional parameters of year of construction and presence of short columns were taken into considerations in this research. Finally $60 \%$ of the structures were classified with high priority. Such a high percentage implies that an important number of children are in high risk if a seismic event takes place.
\end{abstract}

Keywords: Seismic risk, seismic vulnerability, reinforced concrete, schools.

\section{Índice de vulnerabilidade sísmica das escolas da região metropolitana de Medellín, Colômbia}

\section{Resumo}

Este artigo apresenta a avaliação da vulnerabilidade sísmica de um grupo de escolas localizadas nas cidades de Medellín, Itagüí e Sabaneta, Colômbia, utilizando a metodologia do Índice de Prioridade desenvolvida por Hassan e Sözen (1997). O Índice de Prioridade permite a identificação, entre um grupo de estruturas de concreto armado, daqueles que poderiam sofrer danos graves ou colapsos devido a um evento sísmico importante. Uma avaliação de vulnerabilidade sísmica detalhada deve ser aplicada às estruturas identificadas. Um total de 82 estruturas de 30 escolas foram avaliadas neste trabalho. 0 sistema de resistência à carga lateral de todas as estruturas corresponde a armações de concreto armado com enchimento de alvenaria. A aplicação do método do Índice de Prioridade classificou 61\% das estruturas analisadas com alta prioridade, ou seja, existe a possibilidade dessas estruturas sofrerem severos danos de colapso em um evento sísmico. Parâmetros adicionais do ano de construção e presença de colunas curtas foram considerados nesta pesquisa. Finalmente, $60 \%$ das estruturas foram classificadas com alta prioridade. Uma porcentagem tão alta implica que um número importante de crianças está em alto risco se ocorrer um evento sísmico.

Palavras-chave: Risco sísmico, vulnerabilidade sísmica, concreto armado, escolas. 


\section{Introducción}

Un gran porcentaje del territorio Colombiano se encuentra expuesto al riesgo sísmico, el cual es la probabilidad de la ocurrencia de pérdidas (humanas, sociales y económicas) ante la ocurrencia de un sismo. El riesgo depende de la amenaza sísmica, la vulnerabilidad sísmica y la exposición. El 87\% de la población Colombiana se encuentra ubicada en zonas de amenaza sísmica media a alta (AIS, 2010). El crecimiento de la población se concentra en las áreas urbanas del país, lo cual se convierte en una componente de exposición alta. La ciudad de Medellín es la segunda ciudad de Colombia con mayor población después de la capital Colombiana (Bogotá). Medellín cuenta con una población equivalente al $40 \%$ del total de la población del departamento de Antioquia (DANE, 2005) y tiene una amenaza sísmica intermedia.

Es de gran interés reducir o mitigar el riesgo sísmico, para lo cual es necesario realizar una evaluación de la vulnerabilidad sísmica de las estructuras del lugar de interés. Esta evaluación permite identificar las estructuras con capacidad sísmica adecuada y todas aquellas que necesitan algún tipo de intervención para alcanzar una buena resistencia lateral. En esta investigación se utiliza el método del índice prioritario desarrollado por Hassan y Sözen (1997) para clasificar, dentro de un grupo de edificaciones educativas, las estructuras que posiblemente presenten daño severo o colapso en sismos futuros, identificándolas como estructuras prioritarias, es decir, estructuras que requieren de una rápida intervención para asegurar un buen comportamiento en sismos futuros. El método del Índice Prioritario ha sido calibrado después de la ocurrencia de los sismos en Turquía en 1999 (Dönmez y Pujol, 2005) y en Haití en el 2010 (O’brien et al., 2011) y ha sido aplicado después de los sismos en Bingöl en el 2003 y en Wenchuan en el 2008 (Sözen, 2014; Zhou et al., 2013). El Índice Prioritario es un procedimiento que usa información simple la cual puede ser obtenida fácilmente a través de un formato de evaluación en campo para identificar las estructuras más vulne- rables, es decir, aquellas con probabilidad de daño severo o colapso ante la ocurrencia de un evento sísmico y que, por lo tanto, requieren de un estudio de vulnerabilidad sísmica detallado.

Las escuelas son estructuras que han sido afectadas por eventos sísmicos en todo el mundo, y, en algunos casos, se han reportado pérdidas humanas importantes. El daño inducido por un sismo amenaza la vida de estudiantes y profesores; teniendo en cuenta que los estudiantes pasan la mayor parte de su tiempo en las escuelas, la probabilidad de ocurrencia de un sismo es alta mientras los estudiantes se encuentran dentro de estos establecimientos. La ocurrencia de varios sismos alrededor del mundo ha causado daños a escuelas ocasionando la pérdida de vidas humanas (niños): el sismo de Wenchuan (China) ocasionó la pérdida de vida de cerca de 7000 niños y los sismos ocurridos en Spitak (Armenia) y Cachemira (Pakistán) originaron la pérdida de aproximadamente 16000 y 18000 menores respectivamente (UNCRD, 2009).

Las estructuras construidas en Colombia hace más de 30 años tienen una alta probabilidad de ser vulnerables a sismos ya que el primer código sísmico (Código Colombiano de Construcciones Sismo Resistentes (AIS, 1984)) entró en vigencia en el año de 1984. La primera actualización del código sismo Colombiano se denominó Normas Colombianas de Diseño y Construcción Sismo Resistente (AIS, 1998). Este código tuvo cambios significativos con respecto al primer código, superando las deficiencias observadas en cuanto al diseño sísmico después de la ocurrencia de varios sismos en todo el mundo. La última actualización se realizó en el año 2010 (AIS, 2010).

El sismo de Cariaco, Venezuela, que ocurrió en 1997 entre las ciudades Cariaco y Casanay del estado de Sucre, con una magnitud momento $\mathrm{Mw}=6,9$, es otra muestra del riesgo al cual pueden estar sometidos los menores que ocupan los establecimientos educativos. Este sismo provocó colapsos de varias edificaciones de instituciones educativas, entre las cuales se encuentran cuatro edificios escolares pertenecientes a la escuela Valentín Valiente y al Liceo 
Raimundo Martínez Centeno, donde 22 niños y un profesor perdieron sus vidas. Estas dos instituciones se encontraba a 0,47 y $0,92 \mathrm{~km}$ de la falla, con niveles de aceleración estimados en el sitio de 0,53 g (Coronel, 2012). Los edificios colapsados tenían 2 ó 3 pisos y su sistema estructural era de pórticos de concreto reforzado con muros de mampostería. La escuela Valentín Valiente fue construida en 1960 y el Liceo Raimundo Martínez Centeno fue construida en 1970, años donde las estructuras eras construidas con códigos sísmicos que proporcionaban a las estructuras un diseño sísmico bajo a medio de acuerdo a la clasificación presentada por Coronel y López (2012).

El inventario de escuelas del Área Metropolitana de Medellín incluye escuelas de pórticos de hormigón reforzado con muros de mampostería construidas antes de la entrada en vigencia del primer códigos sismo resistente, lo que hace que tengan un nivel bajo a medio de diseño sísmico. El comportamiento de este tipo de estructuras en el sismo de Cariaco permite suponer que las escuelas colombianas tendrían un comportamiento similar si son sometidas a fuerzas sísmicas equivalentes.

Debido a que las escuelas alojan una cantidad alta de menores, cuyas vidas deben protegerse ante la ocurrencia de un sismo, es indispensable evaluar su capacidad sísmica. Idealmente se deberían evaluar detalladamente todas las escuelas de la zona de estudio. Sin embargo, una evaluación detallada requiere de una inversión muy alta de recursos. Es necesario entonces recurrir a una metodología sencilla que permita identificar, de un grupo numeroso de estructuras, aquellas que tienen una menor capacidad sísmica y que, por lo tanto, deben ser consideradas como prioritarias.

En este artículo se presentan los resultados de la evaluación de un inventario de escuelas de Medellín, Itagüí y Sabaneta mediante la aplicación del método del Índice Prioritario. Este estudio permitió priorizar en un periodo corto de tiempo un grupo de 82 edificaciones, identificando aquellas que tienen una mayor vulnerabilidad y que por lo tanto requieren de una evaluación de vulnerabilidad sísmica detallada.

\section{Método del Índice Prioritario}

El índice prioritario fue propuesto por Hassan y Sözen (1997) para edificios de concreto reforzado de baja altura, con niveles bajos en el detallamiento del refuerzo y con la mayoría de los muros de mampostería simple. El índice prioritario se calcula con información simple que se puede obtener en un trabajo de campo: áreas de secciones transversales de elementos estructurales y áreas de piso. Hassan y Sözen (1997) definieron el índice prioritario (PI) como la suma del índice de muro $(W I)$ y el índice de columna $(C D)$ :

$$
P I=W I+C I
$$

El índice de muro es la relación entre el área transversal efectiva de muro en la dirección más débil en la base del edificio y el área total de piso por encima de la base de la estructura. El índice de columna es la relación del área transversal efectiva de columna en la base de la estructura con respecto al área de piso total por encima de la base. Se consideran áreas efectivas el 100\% del área de muros de concreto reforzado, el 10\% del área de muros no reforzados y el 50\% del área de columnas; estos porcentajes representan el aporte de resistencia y rigidez de cada elemento estructural. Las ecuaciones 2 y 3 corresponden a los índices de muro y de columna, donde $\sum A_{C}$ es el área total de sección transversal de columnas en el nivel inferior, $\sum A_{C R}$ representa el área total de sección transversal de muros de concreto reforzado en el nivel inferior en la dirección más débil, $\sum A_{N R}$ representa el área total de sección transversal de muros de mampostería no reforzada en el nivel inferior en la dirección más débil y $\sum A_{P T}$ es el área total de piso por encima de la base del edificio.

$$
W I=\frac{\sum A_{C R}+\frac{\sum A_{N R}}{10}}{A_{P T}} \times 100
$$




$$
C I=\frac{\frac{\sum A_{C}}{2}}{A_{P T}} \times 100
$$

Hassan y Sözen (1997) compararon el índice de muro y el índice de columna para un grupo de edificaciones de pórtico de hormigón reforzado con el nivel de daño registrado en el sismo de Turquía de 1992. Los autores identificaron dos fronteras según el índice prioritario: PI = 0,25 y PI = 0,50. Estas fronteras diferencian tres niveles de daño: daño severo o colapso $(0 \leq \mathrm{PI} \leq 0,25)$, daño moderado $(0,25<\mathrm{PI}$ $\leq 0,50)$ y daño leve o sin daño (PI $\geq 0,50$ ). Debido a la facilidad del cálculo del índice prioritario, este método puede aplicarse de una manera rápida a un inventario numeroso de edificaciones, de modo que se pueda priorizar aquellas estructuras que presentarían mayores daños en el caso de un sismo.

El método del Índice Prioritario se calibró después de la ocurrencia de sismos en Turquía en los años 1992 y 1999, y en Haití en el año 2010 (Hassan y Sözen, 1997; Dönmez y Pujol, 2005; O'brien et al., 2011). La alta concordancia entre los daños esperados según el cálculo del índice prioritario y los daños presentados por los sismos mencionados muestran la efectividad y confiabilidad de la metodología, razón por la cual este método puede usarse para priorizar edificaciones que requieran intervenciones en su sistema de resistencia sísmica. La metodología del Índice Prioritario también fue aplicada después de los sismos ocurri- dos en Bingöl (Turquía) en el año 2003 y en Wenchuan (China) en el año 2008, (Sözen, 2014; Zhou et al., 2013). En Bingöl, de acuerdo a los resultados de los índices prioritarios de 50 edificios y teniendo en cuenta las relaciones entre tipo de daño y valor del índice prioritario propuestas por Hassan y Sözen (1997), se observó que 5 estructuras colapsadas se identificaron correctamente por el método, pero, a su vez, 7 edificios que colapsaron fueron clasificados por el método como estructuras con daño moderado o sin daño. Lo anterior se debe a que el método no incluye la presencia de columnas cortas en la definición del índice prioritario, principal causa del colapso de las estructuras en Bingöl. Como es bien sabido, la presencia de columnas cortas reduce la capacidad sísmica de la estructura. Desafortunadamente la presencia de columnas cortas es común en las escuelas debido al diseño arquitectónico de estas edificaciones, donde la mayoría de las veces se incluyen espacios abiertos amplios para proporcionar la entrada de la luz, dando origen a columnas parcialmente restringidas -columnas cortas-, lo cual hace de la columna un elemento susceptible ante un sismo. De acuerdo a lo anterior es importante tener en cuenta la presencia de columnas cortas en la evaluación de las escuelas. El $67 \%$ de las estructuras evaluadas en el presente estudio tienen columnas cortas, lo cual corresponde a 55 de 82 edificaciones. La Figura 1 presenta algunos ejemplos de columnas cortas en las escuelas evaluadas.

Figura 1. Presencia de columnas cortas en las estructuras evaluadas
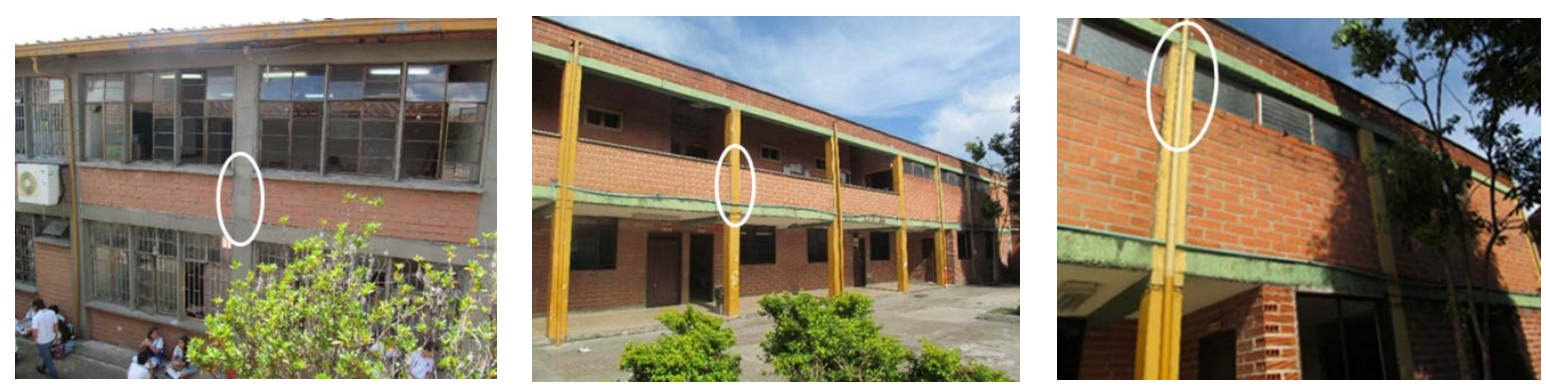


\section{Relación entre el Índice Prioritario}

\section{y la amenaza sísmica en Medellín}

Debido a que el método del Índice Prioritario ha sido definido y calibrado relacionando daños de sismos con las propiedades estructurales de las edificaciones, es necesario identificar si los niveles sísmicos usados en el desarrollo del método son consistentes con la amenaza de Medellín y su Área Metropolitana.

En la Tabla 1 se presentan los parámetros sísmicos (evento, magnitud y profundidad focal) y los parámetros del movimiento sísmico (distancia epicentral, tipo de suelo, velocidad de onda de corte registrada en la estación acelerográfica y la aceleración del terreno) para los sismos ocurridos en: Erzincan (1992), Düzce (1999), Bingöl (2003),
Wenchuan (2008) y Haití (2010), lugares en donde ha sido calibrada y aplicada la metodología del índice prioritario. Todas las aceleraciones presentadas en la Tabla 1 fueron registradas por la estación acelerográfica más cercana a la zona de estudio en donde fue evaluado el método del índice prioritario. Desafortunadamente el sismo ocurrido en Haití en el 2010 no fue registrado por ninguna estación acelerográfica cercana al epicentro; el valor reportado en la Tabla 1 corresponde a un valor sugerido por el U.S. Geological Survey ShakeMap (USGS, 2010) de acuerdo a correlaciones establecidas con niveles de intensidad sísmica. La velocidad de onda de corte más alta, Vs, presentada en la tabla es de $750 \mathrm{~m} / \mathrm{s}$, el cual es un valor más bajo que la velocidad de onda de corte en roca ( $V s \geq 1500 \mathrm{~m} / \mathrm{s}$ ), esto indica que todos los eventos fueron registrados en suelo.

\section{TABLA 1. PARÁMETROS EVENTOS SÍSMICOS - ÍNDICE PRIORITARIO}

\begin{tabular}{|c|c|c|c|c|c|c|}
\hline \multicolumn{3}{|c|}{ Parámetros sísmicos } & \multicolumn{4}{|c|}{ Parámetros del movimiento sísmico } \\
\hline $\begin{array}{l}\text { Evento } \\
\text { sísmico }\end{array}$ & $\begin{array}{l}\text { Magnitud } \\
\text { momento } \\
\qquad \mathrm{Mw}\end{array}$ & $\begin{array}{l}\text { Profundidad } \\
\text { focal } \\
\text { (Km) }\end{array}$ & $\begin{array}{l}\text { Distancia } \\
\text { epicentral } \\
(\mathbf{K m})\end{array}$ & Tipo de suelo & $\begin{array}{c}\text { Vs } \\
\text { Velocidad de onda de } \\
\text { corte }(\mathrm{m} / \mathrm{s})\end{array}$ & $\begin{array}{c}\text { PGA } \\
\text { Aceleración } \\
\text { pico efectiva } \\
\text { (g) }\end{array}$ \\
\hline $\begin{array}{c}\text { Erzincan } \\
1992\end{array}$ & $\begin{array}{l}\text { 6,7 (Sözen, } \\
\text { 2014) }\end{array}$ & 7(USGS, 1992) & $\begin{array}{c}\text { 1,1 (USGS, } \\
1992)\end{array}$ & $\begin{array}{c}\text { Aluvión (Hassan } \\
\text { y Sözen, 1997) }\end{array}$ & 421 (TR-NSMN, 2013) & $\begin{array}{c}0,51 \mathrm{~g} \text { (USGS, } \\
1992)\end{array}$ \\
\hline Düzce 1999 & $\begin{array}{l}\text { 7,2 (Sözen, } \\
\text { 2014) }\end{array}$ & $\begin{array}{c}\text { 13(USGS, } \\
1999)\end{array}$ & $\begin{array}{c}5,9 \text { (USGS, } \\
1999)\end{array}$ & $\begin{array}{l}\text { Suelo blando } \\
\text { (Duzce- } \\
\text { Meteoroloji } \\
\text { station, 2013) }\end{array}$ & 350 (TR-NSMN, 2013) & $\begin{array}{c}\text { 0,51g (USGS, } \\
1999)\end{array}$ \\
\hline Bingöl 2003 & $\begin{array}{c}\text { 6,4 (Sözen, } \\
\text { 2014) }\end{array}$ & $\begin{array}{l}\text { 14(USGS, } \\
2003)\end{array}$ & $\begin{array}{l}\text { 9,1 (USGS, } \\
\text { 2003) }\end{array}$ & $\begin{array}{c}\text { Depósito aluvial } \\
\text { (TUBITAK y NSF, } \\
\text { 2003) }\end{array}$ & $\begin{array}{ll}\text { Tipo C } & 360 \leq V_{s} \leq 750 \\
\text { Tipo D } & 180 \leq V_{s} \leq 360 \\
\text { (TUBITAK y NSF, 2003) }\end{array}$ & $\begin{array}{c}0,52 \mathrm{~g} \text { (USGS, } \\
2003\end{array}$ \\
\hline $\begin{array}{c}\text { Wenchuan } \\
2008\end{array}$ & $\begin{array}{l}\text { 7,9 (Sözen, } \\
\text { 2014) }\end{array}$ & $\begin{array}{l}\text { 19(USGS, } \\
\text { 2008) }\end{array}$ & $\begin{array}{l}\text { 30,0 (Zhou } \\
\text { et al., } \\
2013 \text { ) }\end{array}$ & $\begin{array}{c}\text { Arcilla (Zhou et } \\
\text { al., 2013) }\end{array}$ & 237 (Ren et al., 2012) & $\begin{array}{c}\text { 0,52g (Zhou et } \\
\text { al., 2013) }\end{array}$ \\
\hline $\begin{array}{l}\text { Haití (Port- } \\
\text { au-Prince) }\end{array}$ & $\begin{array}{c}\text { 7,0 (Sözen, } \\
2014 \text { ) }\end{array}$ & $\begin{array}{l}\text { 13(USGS, } \\
2010)\end{array}$ & $\begin{array}{c}16,4 \\
\text { (USGS, } \\
2010)\end{array}$ & $\begin{array}{l}\text { Depósito aluvial } \\
\text { y sedimentario } \\
\text { (O'brien et al., } \\
2011)\end{array}$ & $\begin{array}{c}\text { Tipo } \mathrm{C} \\
360 \leq \mathrm{V}_{\mathrm{s}} \leq 750 \\
\text { Tipo } \mathrm{D} 180 \leq \mathrm{V}_{\mathrm{s}} \leq 360 \mathrm{~m} / \mathrm{s} \\
\text { (O'brien et al., 2011) }\end{array}$ & $\begin{array}{c}0,44 \mathrm{~g} \text { (USGS, } \\
2010)\end{array}$ \\
\hline $\begin{array}{c}\text { Haití } \\
\text { (Léogâne) }\end{array}$ & $\begin{array}{c}7,0 \\
\text { (Sözen, } \\
\text { 2014) }\end{array}$ & $\begin{array}{l}\text { 13(USGS, } \\
2010)\end{array}$ & $\begin{array}{l}\text { 2,4 (USGS, } \\
\text { 2010) }\end{array}$ & - & - & $\begin{array}{c}\text { 0,83g (USGS, } \\
2010)\end{array}$ \\
\hline
\end{tabular}


La aceleración en roca esperada para Medellín, Itagüí y Sabaneta de acuerdo al Código Colombiano Sismo Resistente NSR-10 (AIS, 2010) es 0,15 g para un periodo de retorno de 475 años. Como se mencionó anteriormente, las aceleraciones presentadas en la Tabla 1 fueron registradas en suelo, por lo tanto estas aceleraciones incluyen el efecto amplificador del suelo siendo mayores que las registradas en roca.

De acuerdo al estudio de microzonificación sísmica del Valle de Aburrá (Consorcio Microzonificación 2006, 2007), se esperarían aceleraciones entre 0,2 g y 0,3 g para Medellín; 0,3 g y 0,6 g para Itagüí y 0,3 g y 0,7 g para Sabaneta. Estas aceleraciones incluyen el efecto local del suelo para un periodo de retorno de 475 años. Es importante tener en cuenta que los valores de aceleración indicado en las normas sismo resistentes colombianas y en el estudio de microzonificación están especificados para un periodo de retorno de 475 años, es decir, una probabilidad de excedencia del $10 \%$ en 50 años. Adicionalmente, el valor de aceleración especificado indica que se espera un evento que genere una aceleración mayor o igual a la estipulada. Teniendo en cuenta que las escuelas son estructuras indispensables, la probabilidad de excedencia en 50 años debe ser menor que el $10 \%$ para garantizar un mejor nivel de desempeño; lo anterior implica mayores niveles de aceleración. Si se considerara una probabilidad de excedencia del $5 \%$ en 50 años (equivalente a un periodo de retorno de 975 años), se esperarían aceleraciones en roca para Medellín, Itagüí y Sabaneta iguales a $0,28 \mathrm{~g}, 0,31 \mathrm{~g}$ y $0,32 \mathrm{~g}$ respectivamente (Consorcio Microzonificación 2006, 2007). Al considerar el efecto amplificador del suelo, las aceleraciones esperadas podrían ser aceleraciones superficiales similares o mayores que las registradas en los eventos sísmicos presentados en la Tabla 1.

El estudio de microzonificación indica que los eventos que aportan mayor amenaza a Medellín, Itagüí y Sabaneta, para un periodo de retorno de 475 años, se encuentran ubicados a distancias entre 15 y $20 \mathrm{~km}$ con una magnitud momento $\mathrm{M}_{\mathrm{w}}$ de 6,5. Se observa en la Tabla 1 que el sismo de Bingöl tiene características similares a las definidas por el estudio de microzonificación.

Las aceleraciones esperadas que serían registradas en las escuelas que fueron analizadas en este estudio podrían ser similares, o incluso mayores, a las registradas en los lugares donde el índice prioritario fue calibrado. Se concluye que los escenarios sísmicos con los cuales se ha calibrado el método son consistentes con la amenaza de Medellín y su Área Metropolitana, lo cual le da confiabilidad a la aplicación del método del índice prioritario.

\section{Descripción de los edificios evaluados}

En este estudio se evaluaron 82 estructuras, con un área total de $50815 \mathrm{~m}^{2}$. Las estructuras analizadas pertenecen a instituciones educativas y generalmente son usadas como salones de clase, laboratorios de física y química, aulas de música y deporte, bibliotecas, áreas administrativas y áreas comunes como restaurantes y cocina. Un total de 27 estructuras (33\% del total evaluado) fueron construidas antes de 1984, estas estructuras fueron construidas probablemente sin códigos de resistencia sísmica debido a que el primer código Colombiano se publicó en 1984. Un total de 27 edificios (33\% del total) fueron construidos entre 1984 y 1998, periodo en el que estaba vigente el código sísmico CCCSR-84 (AIS, 1984). El 34\% del total de edificios evaluados fueron construidos después del código sísmico publicado en 1998 (NSR-98).

El trabajo de campo permitió obtener información simple de las escuelas para evaluar el índice prioritario de cada estructura. Adicionalmente, las estructuras se clasificaron teniendo en cuenta las características principales de las edificaciones analizadas como: año de construcción, tipo de cubierta, configuración estructural en planta, número de pisos, características de los elementos no estructurales (muros de mampostería), presencia de columnas cortas. La información obtenida en campo permitió hacer una clasificación de acuerdo a las características estructurales de las escuelas, las cuales se presentan en la Figura 2. 


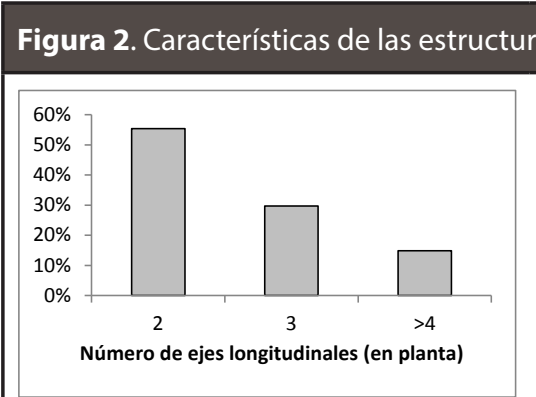

a)

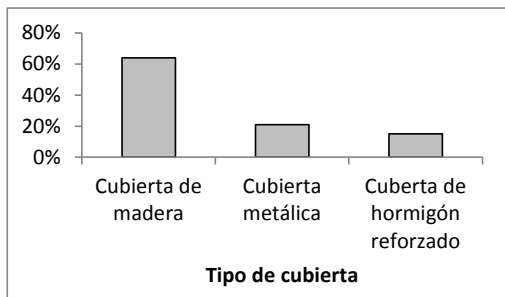

d)

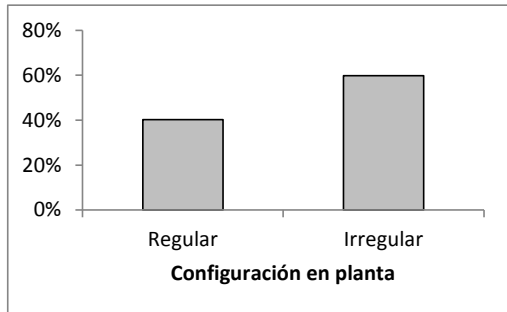

g)

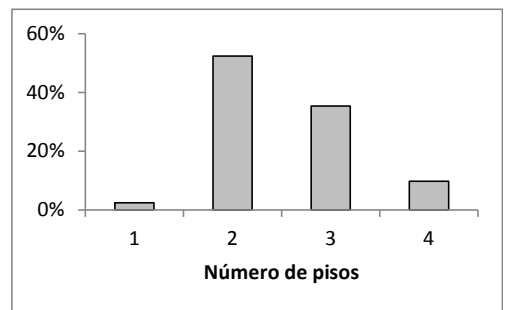

b)

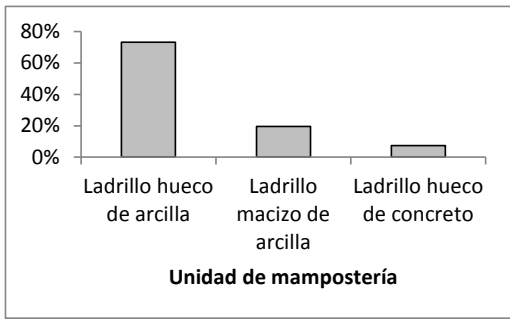

e)

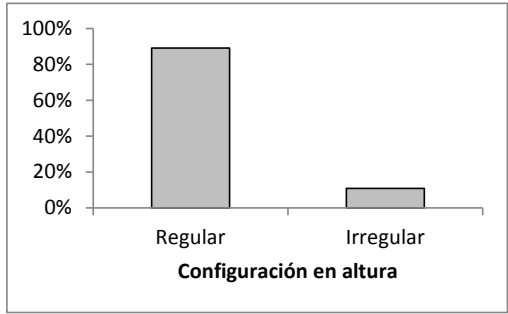

h)

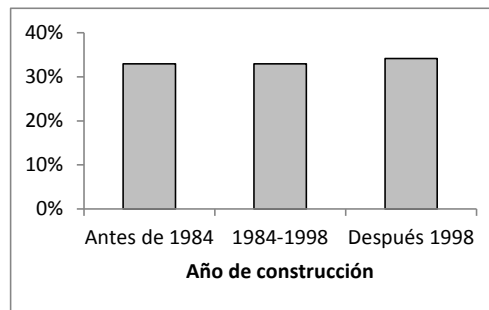

c)

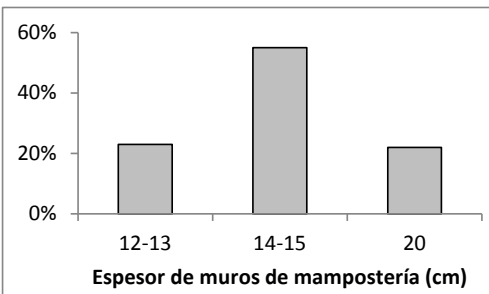

f)

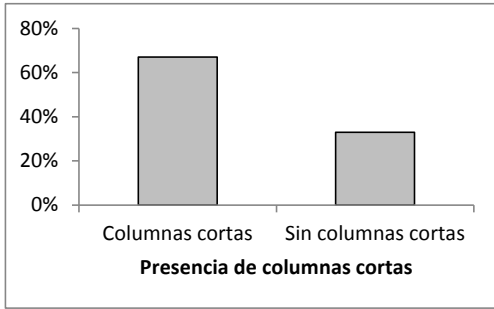

i)

\section{Figura 3. Configuración estructural típica de las escuelas}

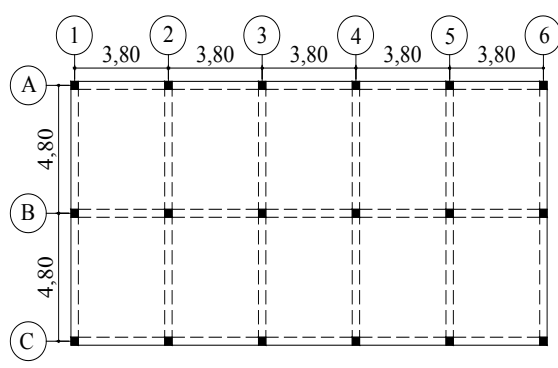

Ejemplo 1-edificio típico (m)

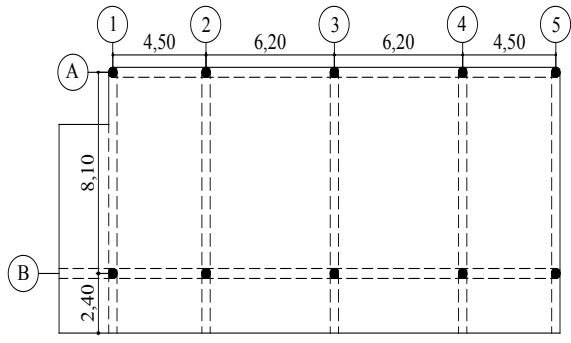

Ejemplo 2-edificio típico (m)

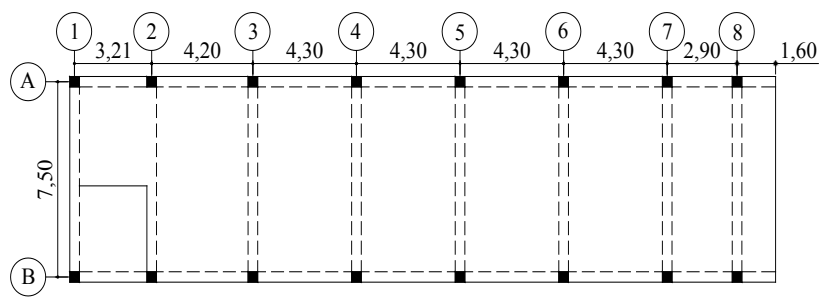

Ejemplo 3-edificio típico (m) 
Figura 4. Irregularidad en planta

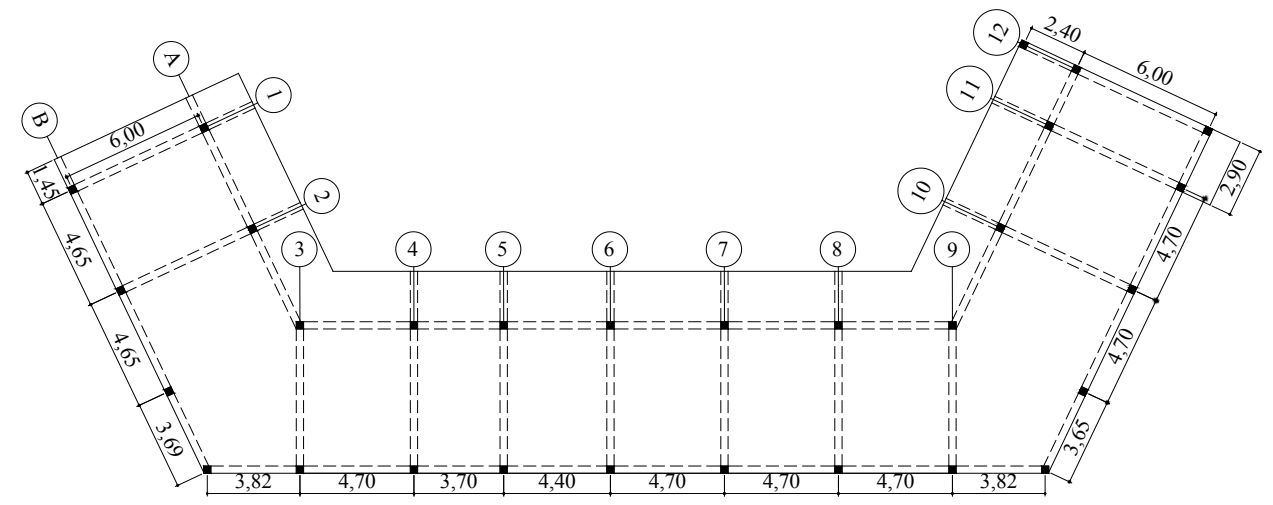

Edificio escolar - planta estructural

\section{Figura 5. Irregularidad en altura}
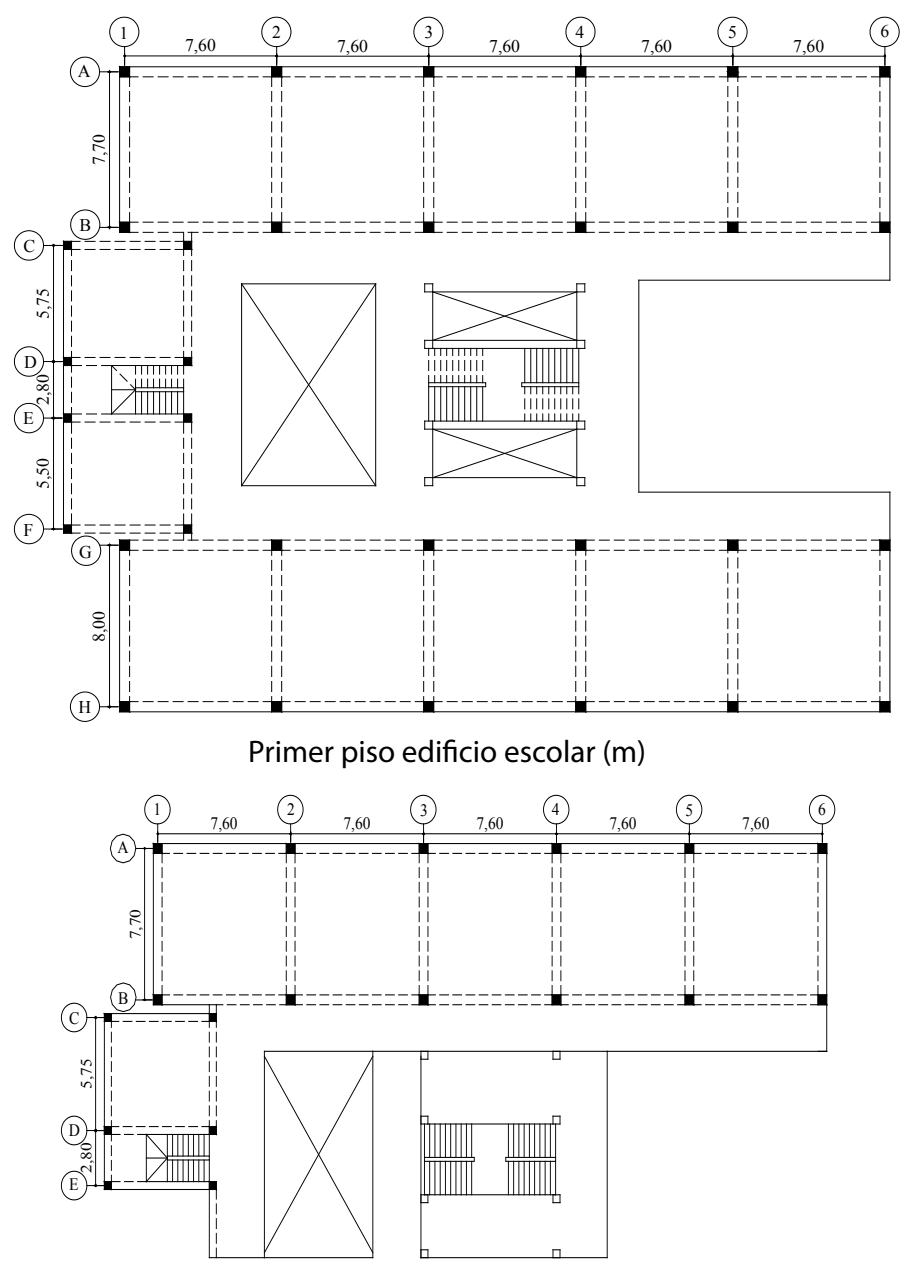

Cuarto piso edificio escolar (m) 
No fue posible identificar el número de ejes longitudinales en planta de 8 estructuras de las 82 edificaciones evaluadas debido a su irregularidad. La configuración estructural típica de las escuelas tiene 2 ejes en la dirección longitudinal en planta con o sin voladizos, lo cual corresponde al 55\% (41 de 74 edificaciones evaluadas). En la Figura 3 se presenta la configuración típica de las escuelas. Se observó que algunos edificios evaluados tienen configuraciones estructurales con irregularidades en planta y en altura. En las Figuras $\mathbf{4}$ y $\mathbf{5}$ se presenta un ejemplo de un edificio con irregularidad en planta y un edificio con irregularidad en altura.

\section{Resultados experimentales y discusión}

El Índice Prioritario define tres niveles de daño de acuerdo al valor del índice de la siguiente manera: daño severo o colapso (PI $\leq 0,25)$, daño moderado $(0,25 \leq \mathrm{PI} \leq 0,50)$ y daño leve o sin daño (PI $>0,50$ ). El $61 \%$ de las estructuras estudiadas tienen una prioridad alta al esperar daño severo o colapso en caso de un evento sísmico. Se espera que el $30,5 \%$ de los edificios analizados tengan un daño moderado mientras que un $8,5 \%$ de los edificios evaluados probablemente estarían sujetos a daño leve o sin daño, lo anterior de acuerdo a lo resultados obtenidos con los cálculos del índice prioritario.

Es importante tener en cuenta que el método del índice prioritario se desarrolló para edificaciones con niveles bajos de detallamiento del refuerzo. El inventario de estructuras analizadas en esta investigación incluye edificaciones construidas de acuerdo a las normas NSR-98 (AIS, 1998) y NSR10 (AIS; 2010), las cuales se espera que tengan un comportamiento sísmico adecuado. Por lo anterior, es necesario considerar el año de construcción de la estructura para evaluar la aplicabilidad del método del índice prioritario. Adicionalmente, se observó en algunas escuelas analizadas presencia de columnas cortas y calidad pobre en la construcción.
Se propuso entonces una nueva clasificación de las estructuras que permitiera tener en cuenta estos aspectos. Se asumió que las estructuras construidas con el código NSR-98 (AIS; 1998) y NSR-10 (AIS, 2010) fueron construidas con un buen nivel de detallamiento sísmico. La Tabla 2 presenta una nueva clasificación, donde se define si la estructura tiene una prioridad alta o no dependiendo del índice prioritario, el año de construcción y la presencia de columnas cortas. De acuerdo a la nueva clasificación se identificaron 32 edificios con una alta prioridad de 50 estructuras con índices prioritarios menores de $0,25 \%$, los cuales fueron construidos con un nivel pobre en el detallamiento del refuerzo (estructuras construidas antes de 1998). Adicionalmente se identificaron 17 estructuras con índices prioritarios mayores de 0,25\% y construidas antes de 1998 con presencia de columnas cortas, para un total de 49 edificios con prioridad alta (60\% de las estructuras evaluadas). Las Figuras 6 y 7 presentan un paralelo entre las dos clasificaciones propuestas, la primera corresponde a la clasificación de Hassan y Sözen (1997) y la segunda corresponde a la clasificación de Hassan y Sözen (1997) teniendo en cuenta el año de construcción y la presencia de columnas cortas.

El paralelo mencionado anteriormente entre las Figuras 6 y 7 muestra que la clasificación propuesta en esta investigación modifica los resultados obtenidos con las fronteras establecidas por Hassan y Sözen (1997). De las edificaciones que quedaron dentro de la frontera 1 propuesta por Hassan y Sözen (1997), 18 de 50 edificaciones resultaron ser estructuras sin prioridad de acuerdo a la clasificación propuesta en la Tabla 2. Igualmente estructuras que clasifican dentro de las zonas con índices prioritarios mayores de $0,25 \%$, lo cual significa que son estructuras para las cuales se esperaría de daño moderado a leve de acuerdo a Hassan y Sözen (1997), resultaron ser edificaciones con prioridad alta (17 de 32 estructuras) de acuerdo a la nueva clasificación propuesta en esta investigación en la Tabla 2. 
TABLA 2. CLASIFICACIÓN ESTRUCTURAL - ÍNDICE PRIORITARIO, AÑO DE CONSTRUCCIÓNY PRESENCIA DE COLUMNAS CORTAS

\begin{tabular}{c|c|c|c}
\multirow{2}{*}{ Prioridad } & Clasificación & Estructuras evaluadas & \% Estructuras evaluadas \\
\hline \multirow{2}{*}{ Prioridad alta } & $A c<1998 I P \leq 0,25$ & 32 & $39,0 \%$ \\
\cline { 2 - 4 } & $A c<1998 I P>0,25$ Cc: Sí & 17 & $21,0 \%$ \\
\hline \multirow{2}{*}{ SUMATORIA PARCIAL } & $A c>1998 I P \leq 0,25$ & $\mathbf{4 9}$ & $\mathbf{6 0 , 0} \%$ \\
\hline \multirow{2}{*}{ Sin Prioridad } & $A c<1998 I P>0,25$ Cc: No & 18 & $22,0 \%$ \\
\cline { 2 - 4 } & $A c>1998 I P>0,25$ & 10 & $6,0 \%$ \\
\cline { 2 - 4 } & SUMATORIA PARCIAL & $\mathbf{3 3}$ & $12,0 \%$ \\
\hline \multicolumn{2}{c|}{ TOTAL } & $\mathbf{8 2}$ & $\mathbf{4 0 , 0} \%$ \\
\hline
\end{tabular}

$A C=$ Año de construcción, $I P=$ Índice prioritario, $C c=$ Columnas cortas

Figura 6. Clasificación estructural - Índice prioritario

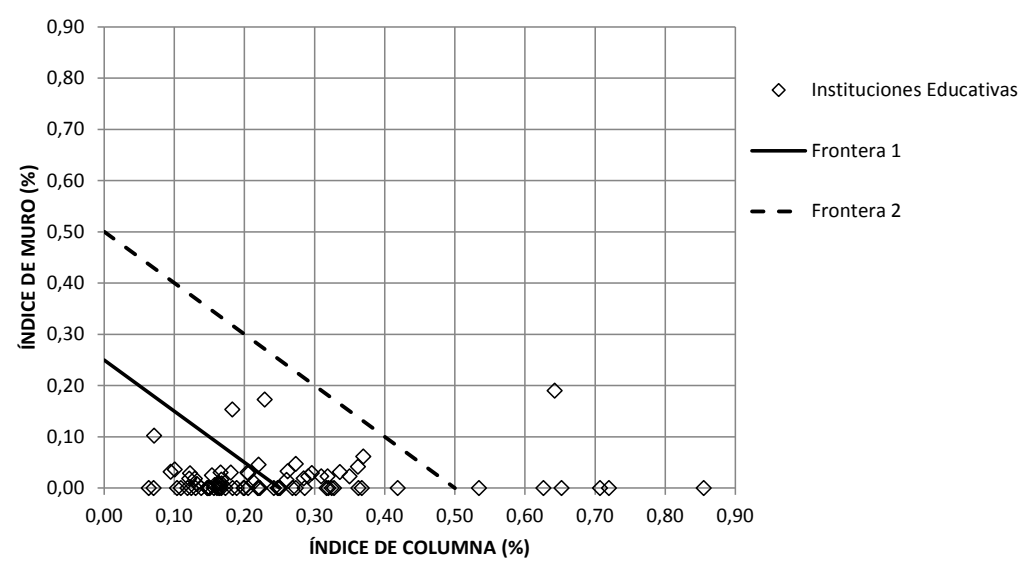

Figura 7. Clasificación estructural - Índice Prioritario, año de construcción y presencia de columnas cortas

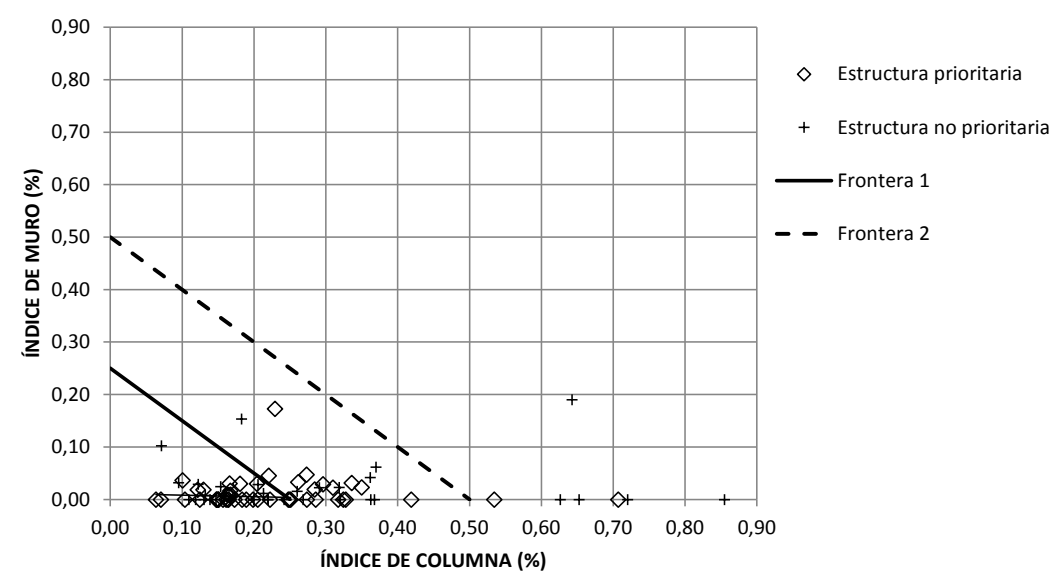




\section{Conclusiones}

En esta investigación se identificó, de acuerdo al método del Índice Prioritario propuesto por Hassan y Sözen (1997), un 61\% de las estructuras evaluadas como estructuras que pueden sufrir daño severo o colapso en caso de un evento sísmico consistente con la amenaza de la región. Lo anterior clasifica estas estructuras como prioritarias, es decir estructuras que requieren rápidamente de una evaluación detallada de vulnerabilidad que permita identificar las medidas necesarias para reducir su vulnerabilidad y, por lo tanto, mitigar el riesgo.

El método del índice prioritario aplicado en esta investigación tiene una desventaja debido a que no identifica la presencia de columnas cortas. Adicionalmente, como el método fue desarrollado a partir de estructuras con niveles bajos y medio de diseño sísmico, es necesario tener en cuenta en la clasificación si la estructura fue diseñada y construida según requisitos sismo resistentes apropiados. Por lo anterior se incluyeron dos criterios adicionales en esta investigación: presencia de columnas cortas y año de construcción; este último asumiendo un buen nivel de detallamiento para las edificaciones construidas después de 1998, año de publicación de la primera actualización del código sísmico Colombiano NSR-98 (AIS, 1998).

De acuerdo a la clasificación presentada en la Tabla 2 se identificaron 49 de 82 estructuras (60\%) con una prioridad alta (estructuras más vulnerables del inventario de edificaciones evaluado), lo que constituye un porcentaje preocupante.

En esta investigación se relacionó la amenaza sísmica de los lugares donde fue calibrado el método del índice prioritario con la amenaza sísmica del Área Metropolitana del Valle de Aburrá y esto permitió identificar que para las ciudades de Medellín, Itagüí y Sabaneta es posible esperar niveles similares de aceleración a los registrados en los lugares donde ha sido evaluado y calibrado el método del índice prioritario, lo que genera confiabilidad en la aplicación del método en el Área Metropolitana de Medellín.
Un número alto de edificaciones fueron identificadas como vulnerables (49 de 82 estructuras), lo cual significa que una gran cantidad de menores se encuentran en riesgo ante la ocurrencia de un evento sísmico. Las estructuras identificadas como prioritarias o con mayor nivel de vulnerabilidad en esta investigación deben ser analizadas con una evaluación de vulnerabilidad sísmica detallada. La información sobre las edificaciones analizadas en este trabajo está disponible para el público en general en la página: https://nees.org/resources/13223/about.

El desarrollo de este trabajo mostró la viabilidad de aplicar el método del índice prioritario al inventario de escuelas de Medellín y su Área Metropolitana. El uso de esta metodología podría utilizarse por entidades gubernamentales para priorizar sus recursos identificando las escuelas que requieren de una evaluación en primer lugar.

\section{Agradecimientos}

Los autores desean expresar su gratitud a la universidad EAFIT por el financiamiento de este trabajo de investigación, al Doctor Santiago Pujol por su asesoría durante el trabajo de investigación y a cada una de las instituciones educativas que permitieron inspeccionar las estructuras.

\section{Referencias}

Asociación Colombiana de Ingeniería Sísmica-AIS, "Reglamento Colombiano de Construcción Sismo Resistente NSR-10", Bogotá: AIS, 2010.

Asociación Colombiana de Ingeniería Sísmica-AIS, “Código Colombiano de Construcción Sismo Resistente CCCSR-84", Bogotá: AIS, 1984.

Asociación Colombiana de Ingeniería Sísmica-AIS, "Reglamento Colombiano de Construcción Sismo Resistente NSR-98", Bogotá: AIS, 1998.

Consorcio Microzonificación 2006, Área Metropolitana del Valle de Aburrá, "Microzonificación y Evaluación del Riesgo Sísmico del Valle de Aburrá”, Medellín: Prográficas Ltda, 184p, 2007.

Coronel, G., "Estimación de Daños y Pérdidas Debidas a Terremotos en Escenarios Regionales: Aplicación a Edificaciones Escolares de Venezuela", Proyecto de 
Grado de Maestría en Ingeniería Sismo resistente, Universidad Central de Venezuela, Caracas, Venezuela 2012.

Coronel, G., López, O.A., "Regional Seismic Damage, Loss and Risk Scenearios of Venezuelan School Buildings", In: Proceedings of the Fifthteenth World Conference on Earthquake Engineering, Lisbon, Portugal, 2012.

Departamento Administrativo Nacional de EstadísticaDANE, “Censo General 2005-República de Colombia, Bogotá, 2005.

Dönmez, C., Pujol, S., "Spatial Distribution of Damage Caused by the 1999 Earthquakes in Turkey", Earthquake Spectra, 21(1): 53-69, 2005.

Hassan, A., Sözen, A., "Seismic Vulnerability Assessment of Low-Rise Buildings in Regions with Infrequent Earthquakes", In: ACI Structural Journal, 94(1): 31-19, 1997.

National strong-motion network of Turkey (TR-NSMN), Duzce-Meteoroloji Mudurlugu Station, (Online), (Cited: 12 nov.2013), <http://www.isesd.hi.is/esd_local/cdrom/reports/SR553.htm>.

National strong-motion network of Turkey (TR-NSMN), Erzincan-Meteorologij Mudurlugu Station, (Online), (Cited: 12 nov.2013), < http://www.isesd.hi.is/ esd_local/cdrom/reports/SR205.htm>.

O'brien, P., Eberhard, M., Haraldsson, O., Irfanoglu, A., Lattanzi, D., Laurer, S., Pujol, S., "Measures of the Seismic Vulnerability of Reinforced Concrete Buildings in Haití", Earthquake Spectra, 27(S1): 373386, 2011.

Ren, Y., Wen, R., Chen, G., “Using Numerical Simulation Via Wave Finite Element Method to Study the Effect of the Station Surrounding on the Strong-Motion Recordings", Institute of Engineering Mechanics, China Earthquake Administration, Harbin, Recent Advances in CSIE 2011, LNEE 126, pp. 353-359, 2012.

Shiga, T., Shibata, A., Takahashi, T., "Earthquake Damage and Wall Index of Reinforced Concrete Buildings", In: Proceedings, Tohuku District Symposium (In Japanese), Architectural Institute of Japan, 12: 29-32, 1968.

Sözen, A., "Surrealism in Facing the Earthquake Risk, In: Seismic Evaluation and Rehabilitation of Structures", 26: 1-13, 2014.

TUBITAK and NSF (teams of engineers), "1 May 2003 Bingöl Earthquake Engineering Report”, Scientific and
Technical Research Council of Turkey (TUBITAK) and U.S. National Science Foundation (NSF), 140p, 2003.

United Nations Centre for Regional Development UNCRD. (2009). Reducing vulnerability of school children to earthquakes. A project of School Earthquake Safety Initiative (SESI). http://www.preventionweb.net/ files/2951_SESIOutcomeallfinal.pdf

U.S. Geological Survey USGS, "13 March 1992 Erzincan", Turkey, Earthquake Shake-Map, Atlas $\mathrm{N}^{\circ}$ 199203131718, 1992, (Online), (Cited:10 oct.2013), <http://earthquake.usgs.gov/earthquakes/shakemap/atlas/shake/199203131718/>.

U.S. Geological Survey USGS, "12 November 1999 Düzce", Turkey, Earthquake Shake-Map, Atlas $\mathrm{N}^{\circ}$ 199911121657, 1999, (Online), (Cited:10 oct.2013), <http://earthquake.usgs.gov/earthquakes/shakemap/atlas/shake/199911121657>.

U.S. Geological Survey USGS, “1 May 2003 Bingöl”, Turkey, Earthquake Shake-Map, Atlas № 200305010027, 2003, (Online), (Cited: 15 oct.2013), <http://earthquake.usgs.gov/earthquakes/shakemap/atlas/ shake/200305010027/>.

U.S. Geological Survey USGS, “12 May 2008 Sichuan”, China, Earthquake Shake-Map, Atlas $N^{\circ}$ us2008ryan, 2010, (Online), (Cited: 15 0ct.2013), <http://earthquake.usgs.gov/earthquakes/shakemap/global/ shake/2008ryan/ >.

U.S. Geological Survey USGS, "12 January 2010 Haiti", Earthquake Shake-Map, Atlas $\mathrm{N}^{\circ}$ us2010rja6, 2008, (Online), (Cited: 20 feb.2013), <http://earthquake.usgs.gov/earthquakes/shakemap/global/ shake/2010rja6/ >

Zhou, W., Zheng, W., Pujol, S., "Seismic Vulnerability of Reinforced Concrete Structures affected by the 2008 Wenchuan Earthquake”, In: <https://nees.org/ warehouse>, 21p, 2013.

\section{PARA CITAR ESTE ARTÍCULO / TO REFERENCE THIS ARTICLE / PARA CITAR ESTE ARTIGO /}

Zora Mejía, F.N.; Acevedo Jaramillo, A.B. (2019). Índice de vulnerabilidad sísmica de escuelas del Área Metropolitana de Medellín, Colombia. Revista EIA, 16(32), Julio-Diciembre, pp. 195-207. [Online]. Disponible en: https://doi.org/10.24050/ reia.v16i32.1035 